\title{
Earth Currents Near a Top-Loaded Monopole Antenna With Special Regard to Electrically Small L-and T-Antennas
}

\author{
H. Lottrup Knudsen
}

\begin{abstract}
An investigation has been made of the ground currents near a top-loaded monopole with nonazimuthal symmetry. Formulas have been developed for the surface current density produced by an inclined, straight wire over a horizontal ground plane for an arbitrary current distribution on the antenna. Working formulas have been developed and numerical calculations of the surface current density on the ground plane have been carried out for the case of a small antenna with a linear current distribution. These results have been used for the calculation of the contribution to the surface current density due to the top loading in the case of an L-antenna and in the case of a T-antenna. In each case both the absolute value of the surface current density arising from the top loading and the relative value of its $\phi$-component have been plotted, as it may be expected that this component under certain circumstances may be important in calculating the ground losses in the case of a system of radial ground wires.
\end{abstract}

\section{Introduction}

Low-frequency and very-low-frequency antennas often consist of a vertical wire with a top loading of inclined, or in a special case, horizontal, wires radiating from the top of the vertical member as shown in figure 1a. In order to reduce the losses in the ground around the antenna, caused by the currents induced in the earth, a system of ground wires is generally used. As the ground currents have essentially a radial direction, it is a common practice to let the ground wires point radially outwards from the base of the antenna.

In the far zone field of the antenna the main contribution to the field comes from the vertical wire. If the top loading is horizontal, the ratio of the con-

J. R. Wait, Earth currents near a monopole antenna with symmetrical top loading, J. Research NBS 62, (1959) R P2959.
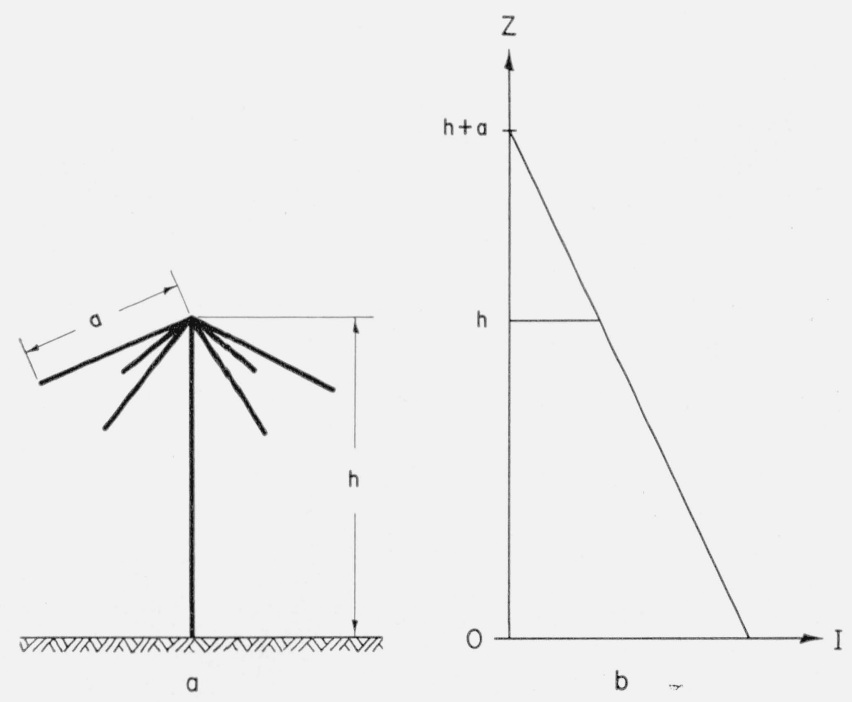

Figure 1. a, Top-loaded antenna. b, Assumed current distribution on this antenna. tribution to the field coming from the horizontal wires to the contribution coming from the vertical wire tends toward zero, as the distance from the antenna goes toward infinity. Also in calculating the ground currents and the ground losses, i.e., in calculating the field at the ground near the antenna, it has generally been assumed without proof, that the contribution from the top loading is insignificant. Recently Wait ${ }^{1}$ has investigated this question. In his investigation he assumes that the top loading may be replaced by a cone, or, in the special case of a horizontal top loading, by a disk; i.e., he assumes perfect rotational symmetry. In his investigation, Wait arrives at the result that under usually existing circumstances the top loading contributes only to a small extent to the currents and losses in the ground.

However, as has been pointed out by Wait, when the number of wires in the top loading is very small, say one, as in the case of the L-antenna, or two, as in the case of the T-antenna, the top loading may furnish a circumferential component of the ground current, which, although small, may contribute considerably to the ground losses, as it is perpendicular to the radial ground wires. It seems, therefore, justified to carry out an investigation of the ground current excited by a top loading consisting of only a few wires and to compare it with the contribution from the vertical wire. This is the object of the present paper. Whereas formulas for the ground current have been worked out for the general case, working formulas and numerical calculations are given only for the case where the antenna is small compared to the wavelength.

The theory has been developed under the assumption that the current distribution on the antenna including that on top loading is known. For antennas that are short compared to the wavelength, we may make the following statements: If the number of wires in the top loading is large, the current distribution will probably be very near quadratic as was assumed by Wait in his investigation of a disk-loaded antenna. On the other hand, if the 
number of top-loading wires is small, say one or two, as we shall assume here, the current distribution will probably be linear, to a good approximation. This is shown in figure $1 \mathrm{~b}$, where $h$ denotes the length of the vertical wire, $a$, the length of the radial wires, (these being assumed to be of equal length), and $I$, the antenna current. A part of the following theory has been derived for an arbitrary current distribution on the antenna. However, in those parts of the investigation where a specific choice of the current distribution has been made, as e.g., in the numerical calculations, the linear current distribution has been chosen.

\section{List of Principal Symbols}

$a=$ length of radial wires in top loading,

$\bar{A}=$ vector potential of current on inclined wire,

$A_{m}=$ coefficient in power series expansion for current distribution function $g(\sigma)$,

$b=$ height over ground plane of one end of inclined wire,

$B=-\frac{\rho}{c} \sin \alpha \cos (\phi-\beta)-\frac{b}{c} \cos \alpha$,

$c=$ length of inclined wire,

$C=\left(\frac{\rho}{c}\right)^{2}+\left(\frac{b}{c}\right)^{2}$,

$d=$ reference distance (arbitrary),

$f(s)=$ current distribution function for inclined wire in terms of $s$,

$g(\sigma)=$ current distribution function for inclined wire in terms of $\sigma$,

$h=$ length of vertical member of antenna,

$I(s)=$ current on inclined wire,

$I_{o}=$ reference current (arbitrary),

$I^{\prime}=$ current in one top-loading wire at connection point with vertical wire,

$I_{b}=$ current in vertical member at ground,

$I_{t}=$ current in vertical member at connection point with top loading,

$\bar{j}=$ normalized surface current density on ground plane: $\frac{d}{I_{0}} \bar{K}$,

$\bar{j}^{h}=$ normalized surface current density on ground plane excited by one horizontal wire,

$\bar{j}^{H}=$ normalized surface current density on ground plane excited by horizontal members of T-antenna,

$\bar{j}^{+}=$normalized surface current density on ground plane excited by horizontal members of antenna with four toploading wires,

$\bar{j}^{0}=$ normalized surface current density on ground plane excited by vertical member of antenna with top loading,

$J=$ the integral $\int_{0}^{c} \frac{f(s) e^{i k \tau}}{r} d s$,

$\bar{K}=$ surface current density on ground plane,

$n=$ number of wires in top loading,
$N=C-B^{2}$,

$p=$ moment of Hertz dipole,

$p_{m}=$ the integral $\int_{0}^{1}\left[\left(\frac{c}{R}\right)^{3}-i k c\left(\frac{c}{R}\right)^{2}\right] \sigma^{m} d \sigma$,

$P=$ the integral $\int_{0}^{1}\left[\left(\frac{c}{R}\right)^{3}-i k c\left(\frac{c}{R}\right)^{2}\right] g(\sigma) e^{i k R} d \sigma$,

$r=$ distance from point of antenna $(\xi, \eta, \zeta)$ to field point $(\rho, \phi, z)$,

$R=$ distance from point of antenna $(\xi, \eta, \zeta)$ to point of ground plane $(\rho, \phi, 0)$,

$s=$ coordinate along inclined wire,

$\hat{s}=$ unit vector pointing in positive direction of inclined wire,

$\hat{s}_{1}, \hat{s}_{2}=$ unit vectors pointing in the two directions of horizontal members of T-antenna,

$S=$ point of penetration of inclined wire through ground plane,

$\hat{x}$ etc. $=$ unit vector in $x$-direction etc.,

$X=\left(\frac{R}{c}\right)^{2}$,

$X_{1}=C+2 B+1$,

$\alpha=$ angle between downward vertical direction (negative z-axis) and positive direction $(\hat{s})$ of inclined wire,

$\beta=$ azimuth of inclined wire,

$v=$ any one of the coordinates $\rho, \phi$ or $z$,

$\rho, \phi, z=$ cylindrical coordinates of field point,

$\sigma=$ normalized coordinate $s / c$ along inclined wire,

$\xi, \eta, \zeta=$ rectangular coordinates of point of inclined wire.

\section{Earth Currents Excited by Inclined Wire}

In this section the earth current excited by the current in an inclined straight wire will be calculated, with special reference to the case where the length of the wire, as well as its height over the ground plane, is small compared to the wavelength.

The ground plane is taken as the $(x, y)$ plane in an $(x, y, z)$ coordinate system. A wire of the length $c$ with one of its ends situated on the z-axis at the height $b$ over the $(x, y)$ plane forms the angle $\alpha$ with this axis as shown in figure 2. A coordinate $s$ along the wire is introduced, $s=0$ corresponding to the point of the z-axis. The wire is assumed to carry a current $I(s)=I_{0} f(s)$ where $I_{0}$ is an arbitrarily chosen reference current. In this section no particular choice of $I_{o}$ is made.

The normalized coordinate $\sigma=s / c$ and the function $g(\sigma)=g(s / c)=f(s)$ are introduced. The antenna may be described in parametric form in the rectangular coordinate system $(x, y, z)$ as follows:

$$
\begin{aligned}
& \xi=s \sin \alpha \cos \beta, \\
& \eta=s \sin \alpha \sin \beta, \\
& \zeta=b-s \cos \alpha,
\end{aligned}
$$




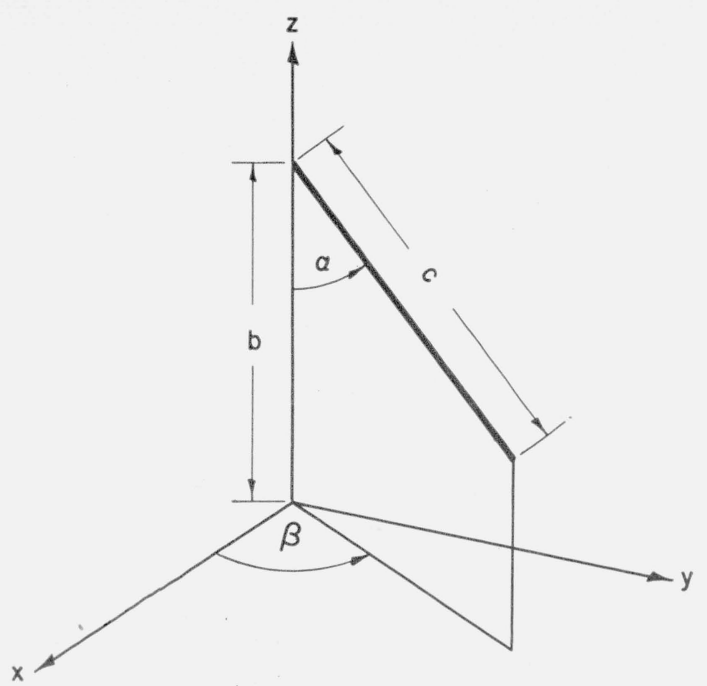

FIGURE 2. Inclined wire above ground plane.

With the time factor $e^{-i \omega t}$ the rectangular coordinates of the vector potential $\overline{\mathrm{A}}$ are expressed by

$$
\begin{aligned}
& A_{x}=\frac{\mu I_{o}}{4 \pi} \sin \alpha \cos \beta J, \\
& A_{y}=\frac{\mu I_{o}}{4 \pi} \sin \alpha \sin \beta J, \\
& A_{2}=-\frac{\mu I_{0}}{4 \pi} \cos \alpha J,
\end{aligned}
$$

where

$$
J=\int_{0}^{c} \frac{f(s) e^{i k r}}{r} d s
$$

with

$r=\left[(s \sin \alpha \cos \beta-\rho \cos \phi)^{2}+(s \sin \alpha \sin \beta\right.$

$$
\left.-\rho \sin \phi)^{2}+(b-s \cos \alpha-z)^{2}\right]^{1 / 2},
$$

where $(\rho, \phi, z)$ denote the cylindrical coordinates of the field point. The coordinates of the vector potential $\bar{A}$ in the cylindrical coordinate system are

$$
A_{\rho}=A_{x} \cos \phi+A_{y} \sin \phi=\frac{\mu I_{o}}{4 \pi} \sin \alpha \cos (\phi-\beta) J,
$$$$
A_{\phi}=-A_{x} \sin \phi+A_{y} \cos \phi=-\frac{\mu I_{o}}{4 \pi} \sin \alpha \sin (\phi-\beta) J,
$$$$
A_{2}=-\frac{\mu I_{0}}{4 \pi} \cos \alpha J
$$

The magnetic field intensity $\bar{H}$ may now be obtained from

$$
\bar{H}=\frac{1}{\mu} \nabla \times \bar{A} .
$$

Until now, the effect of the ground plane has not been considered. Assuming this to have infinite conductivity, this may be done by adding the field of the image. Since we are interested only in the tangential magnetic field at the ground plane, this is twice the tangential component of the magnetic field calculated above.

For the current density $\bar{K}$ in the ground Iplane, then,

$$
\bar{K}=2(\hat{z} \times \bar{H})_{z=0} .
$$

It is convenient to define a dimensionless, "normalized surface current density

$$
\bar{j}=\frac{d}{I_{0}} \bar{K}
$$

where $d$ is an arbitrarily chosen reference distance. With the above definition of $\bar{j}$ it is found that

$$
\begin{aligned}
& j_{\rho}=-\frac{2 d}{I_{o}}\left(H_{\phi}\right)_{z=0}, \\
& j_{\phi}=\frac{2 d}{I_{o}}\left(H_{\rho}\right)_{z=0} .
\end{aligned}
$$

Defining

$$
P=\int_{0}^{1}\left[\left(\frac{c}{R}\right)^{3}-i k c\left(\frac{c}{R}\right)^{2}\right] g(\sigma) e^{i k R} d \sigma,
$$

with

$R=(r)_{z=0}=\left[(s \sin \alpha \cos \beta-\rho \cos \phi)^{2}+(s \sin \alpha \sin \beta\right.$

$$
\begin{array}{r}
\left.-\rho \sin \phi)^{2}+(s \cos \alpha-\mathrm{b})^{2}\right]^{1 / 2} \\
=c\left[\left(\sigma \sin \alpha \cos \beta-\frac{\rho}{c} \cos \phi\right)^{2}+(\sigma \sin \alpha \sin \beta\right. \\
\left.\left.-\frac{\rho}{c} \sin \phi\right)^{2}+\left(\sigma \cos \alpha-\frac{b}{s}\right)^{2}\right]^{1 / 2}(10)
\end{array}
$$

we finally obtain after some manipulation

$$
\begin{aligned}
& j_{\rho}=\frac{1}{2 \pi} \frac{d}{c}\left[-\frac{b}{c} \sin \alpha \cos (\phi-\beta)+\frac{\rho}{c} \cos \alpha\right] P, \\
& j_{\phi}=\frac{1}{2 \pi} \frac{d}{c} \frac{b}{c} \sin \alpha \sin (\phi-\beta) P .
\end{aligned}
$$

The first term in the square bracket in the expression for $P$ corresponds to the induction field, whereas the last term corresponds to the radiation field.

It is easily verified, from the above expressions for the components of $\bar{j}$ as well as by physical considerations, that $j_{\rho}$ is an even function of $\phi-\beta$, whereas $j_{\phi}$ is an odd function of $\phi-\beta$.

As a partial check of the formulas developed here, these formulas may be applied to a short vertical wire with a constant current $I_{o}$ as considered by 
Wait (see footnote 1). This may be done, for example, by setting

$$
\begin{gathered}
b=0, \\
\alpha=\pi, \\
g(\sigma)=1, \\
e^{i k R} \simeq e^{i k_{\rho}} .
\end{gathered}
$$

Then

$$
\begin{aligned}
& K_{\rho}=\frac{I_{0}}{d} j_{\rho}=\frac{I_{o}}{2 \pi} \frac{\rho}{c^{2}} \cos \alpha, P=-\frac{I_{0} e^{i k_{\rho} \rho}}{2 \pi} \int_{0}^{a}\left[\frac{1}{R^{3}}-\frac{i k}{R^{2}}\right] d s \\
& K_{\phi}=0
\end{aligned}
$$

where

$$
R=\left[\rho^{2}+s^{2}\right]^{1 / 2} .
$$

This expression checks with formula (7) in the above mentioned paper by Wait (except for the sign; a different sign convention seems to have been used by Wait).

Whereas it is necessary to evaluate the integral $P$ in order to obtain the normalized surface current density $\bar{j}$, the direction of $\bar{j}$ may be obtained without carrying out any integration. The reason for this is that all the current elements of the wire give contributions to $\bar{j}$ pointing in the same direction. The direction of the current is expressed by the angle $u$ which it forms with the $\rho$-axis, as shown in figure 3 .

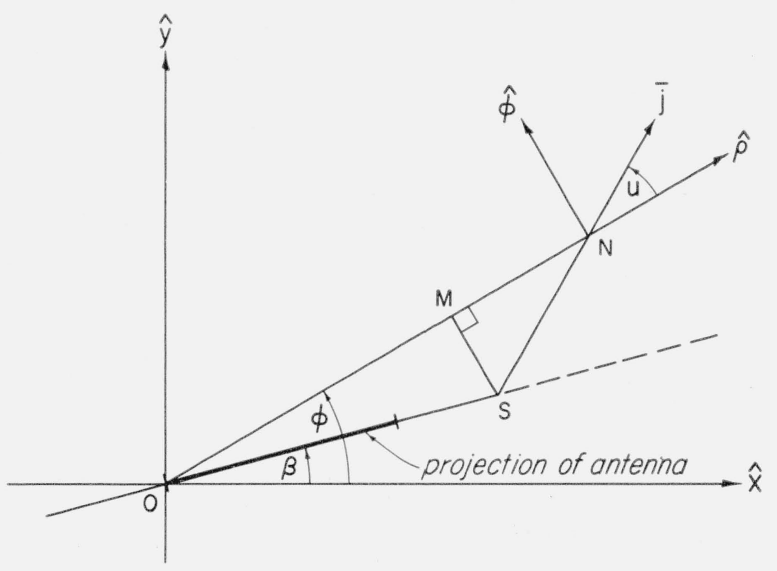

Figure 3. Surface current density.

The surface current density $j$ points radially outward from (or toward) the point of pentration $\mathrm{S}$ through the ground plane of the antenna or its prolongation.

It is found that

$u=\tan ^{-1} \frac{j_{\phi}}{j_{\rho}}=\tan ^{-1} \frac{b \sin \alpha \sin (\phi-\beta)}{-b \sin \alpha \cos (\phi-\beta)+\rho \cos \alpha}$.

It will be shown that this expression exactly expresses that the surface current density $\bar{j}$ at every point of the ground plane points radially outward from (or toward) the point $\mathrm{S}$ in which the inclined wire or its prolongation penetrates the ground plane. Referring again to figure 3 , it is found that the distance from the origin $\mathrm{O}$ to the point $\mathrm{S}$ is $b \tan \alpha$. Introducing the perpendicular SM from $\mathrm{S}$ to the line from the origin $\mathrm{O}$ to the field point $\mathrm{N}$, if the current at $\mathrm{N}$ points radially outward from $\mathrm{S}$, it follows that

$$
u=\tan ^{-1} \frac{\mathrm{SM}}{\mathrm{NM}}=\tan ^{-1} \frac{b \tan \alpha \sin (\phi-\beta)}{\rho-b \tan \alpha \cos (\phi-\beta)} .
$$

As this expression is equal to the expression for $u$ derived above, it is concluded that the surface current density points radially outward from (or toward) the point where the linear antenna or its prolongation penetrates the ground plane. This result, which was arrived at as a detail in a more comprehensive investigation, may of course be derived in a more straightforward manner. In figure $4 \mathrm{a}$ is shown an antenna that penetrates the ground plane at $\mathrm{S}$, and lines that are everywhere parallel to the surface current density. They are not field lines in a strict sense, as their mutual distance is not proportional to the magnitude of the surface current density. In the case where the antenna is parallel to the ground plane, i.e., where the point of penetration is at infinity, the surface current density will be everywhere parallel to the wire. This is illustrated in figure $4 \mathrm{~b}$.
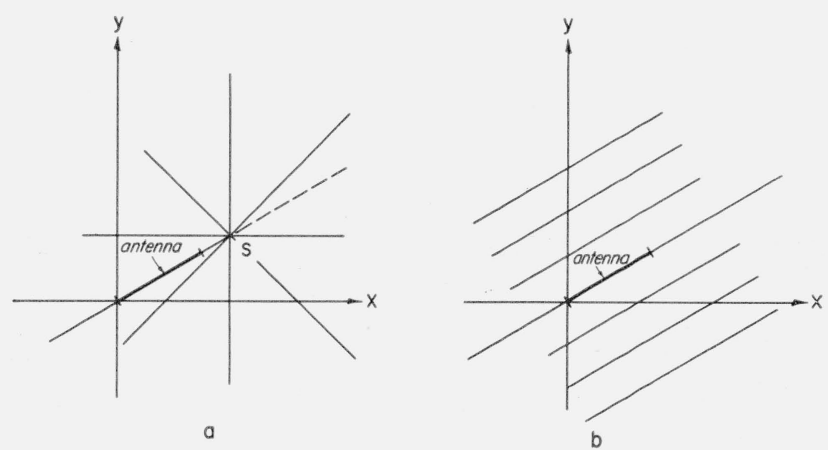

FIgURE 4. Lines showing direction of the surface current density on the ground plane due to a linear antenna.

$\mathrm{a}$, The antenna is not parallel to the ground plane; $\mathrm{S}$ denotes the point of penetration of the antenna through the ground plane. $b$, The antenna is parallel to the ground plane, i.e., the point of penetration is at infinity.

Returning to the calculation of the magnitude of the surface current density, the expression $P$ must be evaluated. In the general case the integral by which $P$ is defined can apparently not be expressed by known functions. In the general case, therefore, numerical or graphical methods must be applied to evaluate $P$. This can be done in any specified case without a prohibitive amount of labor.

A case which has a great practical importance and in which the analysis is greatly simplified is the one where both the height $b$ and the length $c$ of the antenna are small compared to the wavelength or more exactly where

$$
k(b+c)<<1 \text {. }
$$


A similar approximation was used by Wait (see footnote 1) in the case of a vertical antenna. For an inclined wire, as is considered here, this approximation will, for the directions perpendicular to the wire, be just as good as for the vertical wire. However, in the directions of the projection of the wire on the ground plane, the approximation will be worse than in the above mentioned case. Or otherwise expressed, for the directions not perpendicular to the wire, $k c$ must be smaller than for a vertical wire in order that we shall obtain the same approximation.

The rest of this report will be concerned exclusively with this case. Under the above assumption, any case will be included in at least one of the following four situations :

1. $k \rho<<1$,

2. Neither $k \rho<<1$ nor $b+c<<\rho$,

3. $b+c<<\rho$ but not $1<<k \rho$,

4. $1<<k \rho$.

When $k(b+c)$ is not much smaller than 1 , say $k(b+c)=0.2$, situation 2 may apply for some values of $\rho$. On the other hand, if $k(b+c)<<<1$, situations 1 and 3 may apply simultaneously for some values of $\rho$. The above discussion is illustrated in figures $5 \mathrm{a}$ and $5 \mathrm{~b}$ applying respectively to the situation where $k(b+c)$ is relatively large (although conforming to the inequality $k(b+c)<<1)$ and to the situation where $k(b+c)$ is extremely small, $k(b+c)$ $<<<1$.
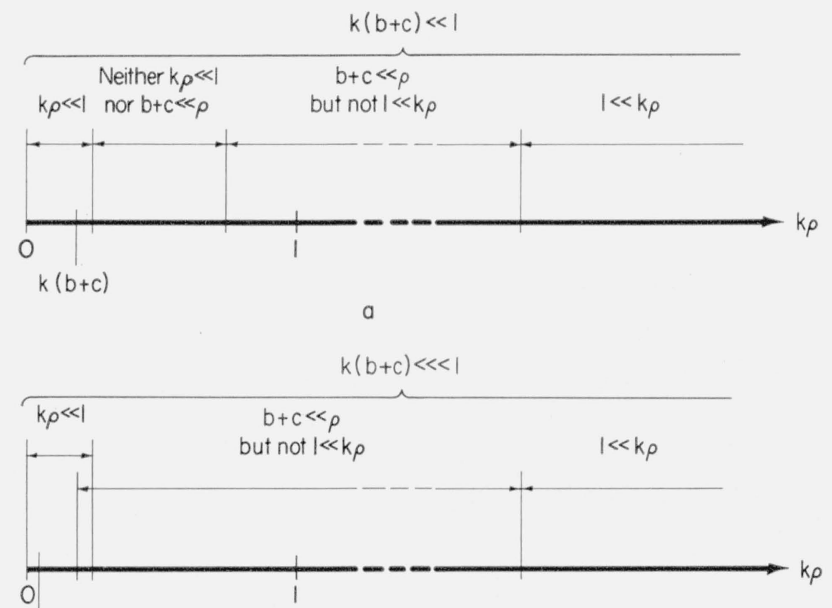

$k(b+c)$

b

Figure 5. Relative position of the various regions of parameters in which various approximations to the integral $\mathrm{P}$ apply.

a, $k(b+c)<<1$, but not extremely small; b, $k(b+c)<<<1$.

$$
\begin{aligned}
k \rho< & <1 \\
P & \simeq \int_{0}^{1}\left(\frac{c}{R}\right)^{3}[1-i k R]\left[1+i k R+\frac{(i k R)^{2}}{2}\right] g(\sigma) d \sigma \\
& \simeq \int_{0}^{1}\left(\frac{c}{R}\right)^{3}\left[1+\frac{(k R)^{2}}{2}\right] g(\sigma) d \sigma \cong \int_{0}^{1}\left(\frac{c}{R}\right)^{3} g(\sigma) d \sigma \\
& + \text { terms containing }(k a)^{2},(k h)^{2},(k \rho)^{2}, \text { etc. }
\end{aligned}
$$

In using the last approximation the radiation field term, as well as the retardation, is neglected.

Neither $k \rho<<1$ nor $b+c<<\rho$

It turns out that the last one of the approximate formulas worked out for the case of $k \rho<<1$ is a special case of the approximate formula in the case of $b+c<<\rho$. Probably, the last formula may therefore be applied with a fair approximation in the present case, too.

$b+c<<\rho$ but not $1<<k \rho$

$$
\begin{gathered}
R=\rho\left[1-2 \frac{s}{\rho} \sin \alpha \cos (\phi-\beta)+\left(\frac{s}{\rho}\right)^{2}-2 \frac{s}{\rho} \frac{b}{\rho} \cos \alpha\right. \\
\left.+\left(\frac{b}{\rho}\right)^{2}\right]^{1 / 2} \cong \rho-s \sin \alpha \cos (\phi-\beta)
\end{gathered}
$$

+ terms containing $\rho\left(\frac{s}{\rho}\right)^{2}, \rho \frac{s}{\rho} \frac{b}{\rho}, \rho\left(\frac{b}{\rho}\right)^{2}$ etc.

$P \cong e^{i k_{\rho}} \int_{0}^{1}\left(\frac{c}{R}\right)^{3}[1-i k R][1-i k c \sigma \sin \alpha \cos (\phi-\beta)] g(\sigma) d \sigma$

$$
+ \text { terms containing } k c\left(\frac{c}{\rho}\right)^{4}, k c \frac{b}{\rho}\left(\frac{c}{\rho}\right)^{3} \text { etc. }
$$

Though this integral may be expressed by known functions for some current distribution functions $g(\sigma)$, those functions which will be used later in this report will, except in the case of $g(\sigma)=$ constant, require rather complicated computations. As $k c<<1$ has been assumed, the term in the last bracket, containing $k c$, may be neglected with a fair approximation. Thus,

$$
\begin{aligned}
P \cong e^{i k_{\rho}} \int_{0}^{1}\left[\left(\frac{c}{R}\right)^{3}-i k c\left(\frac{c}{R}\right)^{2}\right] g(\sigma) d \sigma \\
\quad+\text { terms containing } k c\left(\frac{c}{\rho}\right)^{3} \text { etc. }
\end{aligned}
$$

When using this approximation, the retardation along the antenna is neglected (but not from the antenna to the field point). 
$1<<k \rho$

Here,

from which

$$
k R>>1,
$$

$P \cong-e^{i k_{\rho}} i k c \int_{0}^{1}\left(\frac{c}{R}\right)^{2}[1-i k c \sigma \sin \alpha \cos (\phi-\beta)] g(\sigma) d \sigma$

$$
+ \text { terms containing }(k R)^{-1} \text { etc. }
$$

Using this approximation means to neglect the induction field term. Also in this case it will generally involve too cumbersome calculations to include the second term in the square bracket; as in the case considered above, with a fair approximation this term may be omitted. This means neglecting the retardation along the antenna itself. Then,

$$
P \cong-e^{i k_{\rho}} i k c \int_{0}^{1}\left(\frac{c}{R}\right)^{2} g(\sigma) d \sigma .
$$

In what follows, eq (16), (19), and (21) will be used as working formulas. The first and the third formulas are both special cases of the second formula. Therefore, the following work will be based upon formula (19), and specializations to the two other cases will be made only at the end of this section.

As was done by Wait (see footnote 1) in treating a disk-loaded monopole, it may profitably be assumed here that the distribution function $g(\sigma)$ of the antenna current is expressed by the series

$$
g(\sigma)=\sum_{m=0}^{\infty} A_{m} \sigma^{m}
$$

Inserting the above series expression for the current distribution function $g(\sigma)$ in the integral expressing $P$,

$$
P=e^{i k_{\rho}} \sum_{m=0}^{\infty} A_{m} p_{m}
$$

where

$$
p_{m}=\int_{0}^{1}\left[\left(\frac{c}{R}\right)^{3}-i k c\left(\frac{c}{R}\right)^{2}\right] \sigma^{m} d \sigma .
$$

This integral may be expressed by known functions for any integral value of $m$. The coefficients $p_{m}$ necessary for dealing with a linear current distribution, i.e., $p_{0}$ and $p_{1}$, will be evaluated here.

In order to carry out the integrations, $X=(R / C)^{2}$ is put into the standard form,

$$
X=C+2 B \sigma+\sigma^{2},
$$

where

$$
\begin{aligned}
& B=-\frac{\rho}{c} \sin \alpha \cos (\phi-\beta)-\frac{b}{c} \cos \alpha, \\
& C=\left(\frac{\rho}{c}\right)^{2}+\left(\frac{b}{c}\right)^{2} .
\end{aligned}
$$

In carrying out the integrations it is important to know the sign of $N=C-B^{2}$. It is found that

$$
N=\left(\frac{\rho}{c}\right)^{2}+\left(\frac{b}{c}\right)^{2}-\left(\gamma \frac{\rho}{c}+\delta \frac{b}{c}\right)^{2},
$$

where

$$
\begin{aligned}
& \gamma=\sin \alpha \cos (\phi-\beta), \\
& \delta=\cos \alpha .
\end{aligned}
$$

As

$$
\gamma^{2}+\delta^{2} \leqq 1,
$$

it may easily be verified that

Further defining

$$
N \geqq 0 .
$$

$$
X_{1}=(X)_{\sigma=1}=C+2 B+1,
$$

the following is obtained:

$$
\begin{gathered}
p_{0}=\frac{1}{N}\left[\frac{B+1}{\sqrt{X_{1}}}-\frac{B}{\sqrt{C}}\right]-\frac{i k c}{\sqrt{N}}\left[\tan ^{-1} \frac{B+1}{\sqrt{N}}-\tan ^{-1} \frac{B}{\sqrt{N}}\right] \\
p_{1}=\frac{1}{N}\left[-\frac{B+C}{\sqrt{X_{1}}}+\sqrt{C}\right]-i k c\left[\frac{1}{2} \ln \frac{X_{1}}{C}\right. \\
\left.-\frac{B}{\sqrt{N}}\left(\tan ^{-1} \frac{B+1}{\sqrt{N}}-\tan ^{-1} \frac{B}{\sqrt{N}}\right)\right]
\end{gathered}
$$

These expressions are sufficient for calculating the contributions to the surface current density corresponding to the terms containing $\sigma^{0}$ and $\sigma^{1}$ in the series expression for the current distribution $g(\sigma)$ of the wire. The terms $p_{2}, p_{3}$, etc. corresponding to the higher order terms in the series expression for $g(\sigma)$ may be found for example by using partial integration, but the calculation is tedious. In the following sections there is a use only for the terms $p_{0}$ and $p_{1}$, corresponding to a linear current distribution. The calculation of $p_{1}, p_{2}$, etc., is therefore omitted.

Following the discussion given earlier, simplified expressions will be given here for $p_{0}$ and $p_{1}$ applying when $p$ is respectively very small and very large compared to the wavelength.

$k \rho<<1$

$$
\begin{aligned}
& p_{0}=\frac{1}{N}\left[\frac{1+B}{\sqrt{X_{1}}}-\frac{B}{\sqrt{C}}\right] \\
& p_{1}=\frac{1}{N}\left[-\frac{B+C}{\sqrt{X_{1}}}+\sqrt{C}\right]
\end{aligned}
$$

$1<<k \rho$

$$
\begin{aligned}
& p_{0}=-i k c\left(\frac{\rho}{c}\right)^{-2} \\
& p_{1}=-\frac{i k c}{2}\left(\frac{\rho}{c}\right)^{-2} .
\end{aligned}
$$




\section{Vertical Wire}

The main purpose of this paper is to calculate the earth current excited by a top loading consisting of one or more inclined or, as a special case, horizontal wires. However, a comparison of the contribution to the earth current from the top loading with the contribution from the vertical member of the antenna is desired. The formulas for calculating this contribution may be obtained as a special case of the formulas developed above.

Considering an antenna of the height $h$ and with a few top-loading wires of the length $a$, and assuming therefore a linear current distribution, the current I in the vertical member of the antenna may be expressed by

$$
I=I_{b}\left(1-\frac{s}{a+h}\right)
$$

where $I_{b}$ is the current at the bottom of the vertical wire, and where $s=z$. In this section and the following sections $I_{b}$ will be used as the reference current $I_{o}$ and the height $h$ of the antenna as the reference distance $d$ occurring in the above formulas. In using these formulas on the vertical member it is further set that

$$
\begin{aligned}
& b=0, \\
& c=h, \\
& \alpha=\pi .
\end{aligned}
$$

The distribution function $g(\sigma)$ for the current on the vertical wire is then expressed by

$$
g(\sigma)=1-\frac{h}{a+h} \sigma .
$$

The following expression for the normalized earth current $\bar{j}^{v}$ due to the vertical wire is hereby obtained:

$$
\begin{gathered}
j_{\rho}^{v}=-\frac{1}{2 \pi} \frac{\rho}{h} P, \\
j_{\phi}^{v}=0,
\end{gathered}
$$

where

$$
P=\left(A_{0} p_{0}+A_{1} p_{1}\right) e^{i k_{\rho}}
$$

with

$$
\begin{aligned}
& A_{0}=1, \\
& A_{1}=-\frac{1}{1+\frac{a}{h}}, \\
& p_{0}=\frac{1}{\left(\frac{\rho}{h}\right)^{2} \sqrt{1+\left(\frac{\rho}{h}\right)^{2}}-\frac{i k h}{\frac{\rho}{h}} \tan ^{-1} \frac{1}{\frac{\rho}{h}}}
\end{aligned}
$$

$$
p_{1}=-\frac{1}{\sqrt{1+\left(\frac{\rho}{h}\right)^{2}}}+\frac{1}{\frac{\rho}{h}}-\frac{i k h}{2} \ln \frac{1+\left(\frac{\rho}{h}\right)^{2}}{\left(\frac{\rho}{h}\right)^{2}} .
$$

As the main purpose of this report is to investigate the ground current induced by the top loading, whereas the ground current induced by the vertical member is only calculated for comparison, it may be assumed, for the sake of simplicity, that the vertical wire carries a constant current $I_{1}$ equal to the mean value of the actual current distribution, i.e.,

$$
I_{1}=I_{b}\left[1-\frac{1}{2\left(1+\frac{a}{h}\right)}\right] \text {. }
$$

We then have

$$
P=A_{0} p_{0},
$$

where

$$
A_{0}=1-\frac{1}{2\left(1+\frac{a}{h}\right)}
$$

and where $p_{0}$ is expressed as above. The final expressions for the components of $\bar{j}^{v}$ then become

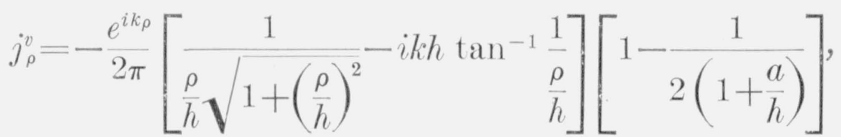

$j_{\phi}^{v}=0$.

These expressions are equivalent to formula (7) in the paper by Wait (see footnote 1) referred to above.

In figure $6\left|j_{\rho}^{v}\right|$ has been plotted for $k h=0$ as a function of $\rho / h$ and with $a / h$ as a parameter. In this case, the last term in the first square bracket in the formula expressing $j_{\rho}^{v}$ drops out. In the case of very large distances $\rho$, however, this last term may become significant and even dominant. Figure 7 shows a similar plot of $\left|j_{\rho}^{v}\right|$ for $k h=0.1$. This value of $k h$ corresponds approximately to the antenna for the planned U.S. Navy vlf radio station, Cutler, Maine (approximate data: $f \cong 15 \mathrm{kc}, h \cong 800 \mathrm{ft}$ ).

\section{Top Loading}

The ground current excited by an umbrella type of top loading may be calculated by adding vectorially the currents excited by the single wires. In this section an expression is given for the surface current density $\bar{j}$ induced by a single wire of the top loading. Letting $\sigma=0$ correspond to that end of the wire under consideration which is connected to the vertical member of the antenna and $\sigma=1$ to the outer end of the wire, the fact that the current at the end of the antenna is zero is expressed by

$$
g(1)=0 .
$$




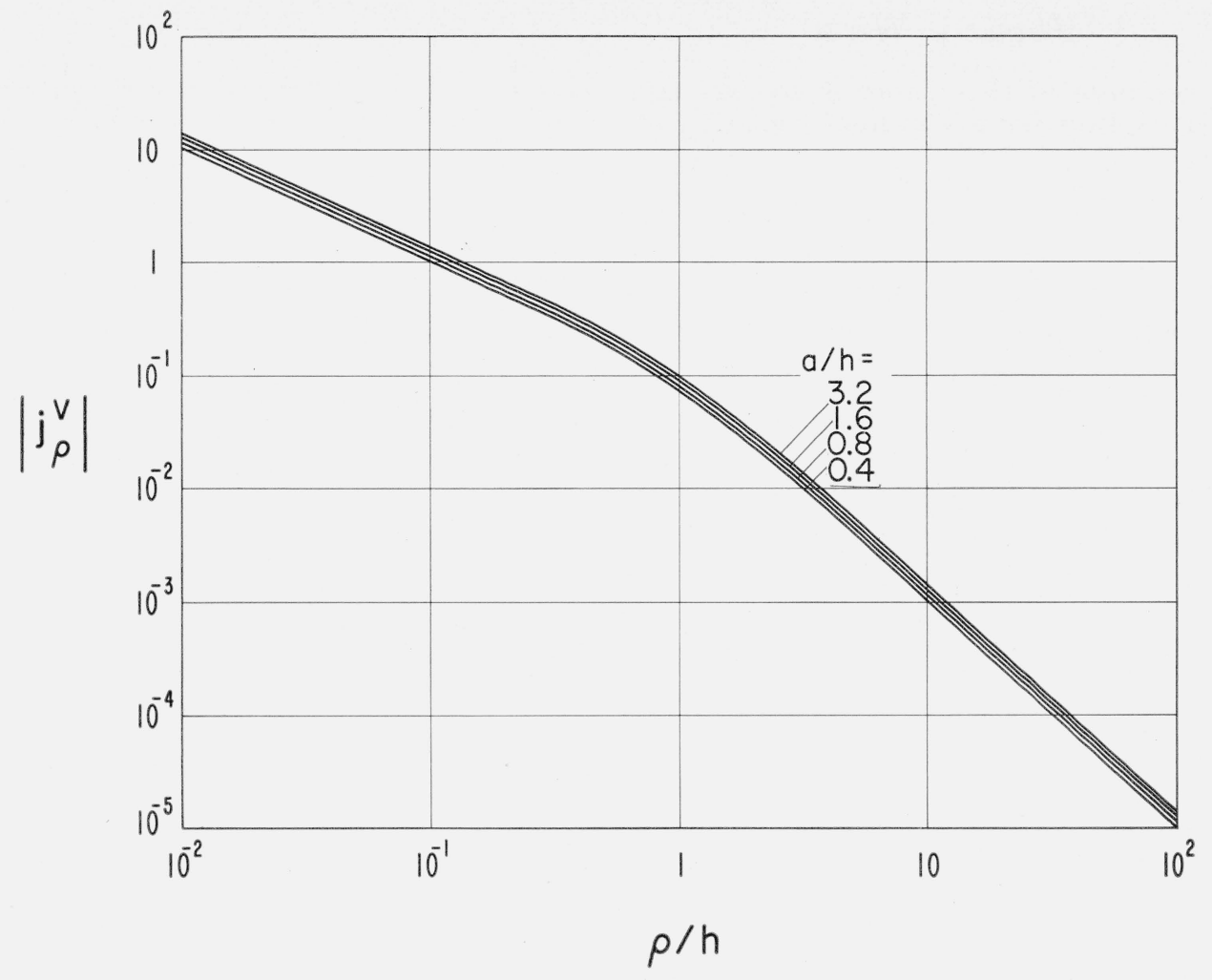

Figure 6. Normalized surface current density $\left|j_{\rho}^{v}\right|$ at the ground plane due to the current in the vertical member of a top-loaded antenna as a function of the normalized distance $\rho / h$ from the base of the antenna and with the normalized length a/h of the top-loading wires as a parameter.

It is assumed that the height $h$ of the antenna is infinitely small compared to the wavelength, i.e., $k h=0$.

Using the series expression introduced above for the current in the antenna, it is found that

$$
\sum_{m=0}^{\infty} A_{m}=0 \text {. }
$$

The current at $\sigma=0$ is denoted by $I^{\prime}$. As in the last section, the current $I_{b}$ at the bottom of the vertical wire will be used as the references current, $I_{o}$. Thus,

$$
g(0)=\frac{I^{\prime}}{I_{b}}
$$

In what follows, it will be assumed that the top loading consists of $n$ equiangularly spaced wires. Each wire will then carry the same current and consequently,

$$
g(0)=\frac{1}{n} \frac{I_{t}}{I_{b}}
$$

where $I_{t}$ is the current at the top of the vertical wire. Using the series expansion for $g(\sigma)$,

$$
A_{0}=\frac{1}{n} \frac{I_{t}}{I_{b}}
$$

In the case of a top loading consisting of one wire (if the wire is horizontal, an L-antenna), or of two wires (if the wires are horizontal, a T-antenna), the current on these wires will probably, to a good approximation, be linear. Then,

$$
A_{0}=-A_{1}=\frac{1}{n} \frac{\frac{a}{h}}{1+\frac{a}{h}}
$$

and all other coefficients $A_{m}$ equal to zero.

On the other hand, if the top loading is of the umbrella type, i.e., if it consists of several equiangularly spaced wires, the current on the wires will probably be better approximated by a quadratic function. In this case,

$$
A_{0}=-A_{2}=\frac{1}{n} \frac{\frac{a}{h}}{1+\frac{a}{h}}
$$

and all other coefficients $A_{m}$ equal to zero. The remaining part of this report will be concerned mainly with the cases of a top loading consisting of 


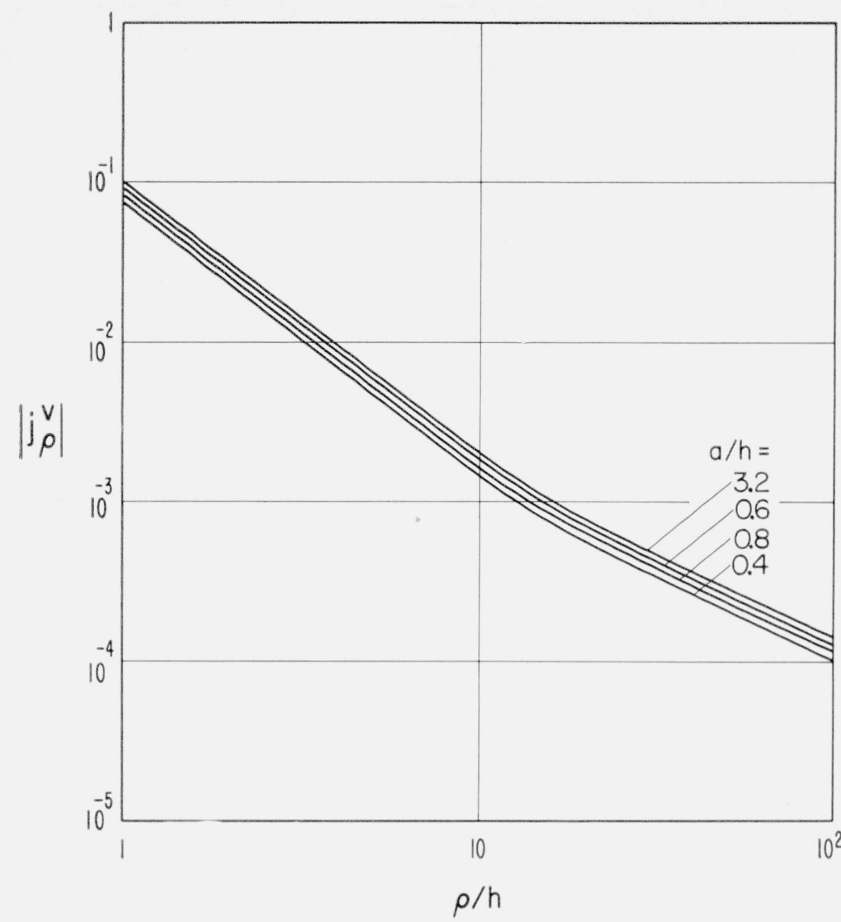

Figure 7. Normalized surface current density $\left|j_{\rho}^{v}\right|$ at the ground plane due to the current in the vertical member of a top-loaded antenna as a function of the normalized distance $\rho / h$ from the base of the antenna and with the normalized length $a / h$ of the top-loading wires as a parameter, the height $\mathrm{h}$ of the antenna being given by $\mathrm{kh}=0.1$.

one $(n=1)$ or two $(n=2)$ wires and the current distribution will consequently be assumed to be linear.

As in the last section, $h$ will be used as the reference distance $d$ in calculating the normalized surface current density. Further setting

$$
\begin{aligned}
& b=h \\
& c=a
\end{aligned}
$$

the following is obtained from the formulas developed above

$$
\begin{aligned}
& j_{\rho}=\frac{1}{2 \pi} \frac{h}{a}\left[-\frac{h}{a} \sin \alpha \cos (\phi-\beta)+\frac{\rho}{a} \cos \alpha\right] P, \\
& j_{\phi}=\frac{1}{2 \pi}\left(\frac{h}{a}\right)^{2} \sin \alpha \sin (\phi-\beta) P,
\end{aligned}
$$

where

$$
\begin{array}{r}
P=\frac{1}{n} \frac{\frac{a}{h}}{1+\frac{a}{h}}\left(p_{0}-p_{1}\right) e^{i k \rho}=\frac{1}{n} \frac{\frac{a}{h}}{1+\frac{a}{h}}\left\{\frac { 1 } { N } \left[\sqrt{X_{1}}-\frac{B}{\sqrt{C}}\right.\right. \\
-\sqrt{C}]+i k h \frac{a}{h}\left[\frac{1}{2} \ln \frac{X_{1}}{C}-\frac{B+C}{\sqrt{N}}\left(\tan ^{-1} \frac{B+1}{\sqrt{N}}\right.\right. \\
\left.\left.\left.-\tan ^{-1} \frac{B}{\sqrt{N}}\right)\right]\right\} e^{i k_{\rho}},
\end{array}
$$

$$
\begin{aligned}
B & =-\frac{\rho}{a} \sin \alpha \cos (\phi-\beta)-\frac{h}{a} \cos \alpha, \\
C & =\left(\frac{\rho}{a}\right)^{2}+\left(\frac{h}{a}\right)^{2}, \\
N & =C-B^{2}, \\
X_{1} & =C+2 B+1 .
\end{aligned}
$$

For numerical calculations, it is of practical importance to have simplified expressions for $P$ valid when $\rho$ is very small and very large compared to the wavelength respectively. Making use of the discussion given earlier, the following is obtained

$k \rho<<1$

$$
P=\frac{1}{n} \frac{\frac{a}{h}}{1+\frac{a}{h}} \frac{1}{N}\left[\sqrt{X_{1}}-\frac{B}{\sqrt{C}}-\sqrt{C}\right]
$$

$1<<k \rho$

$$
\begin{gathered}
P=\frac{1}{2 n} \frac{\frac{a}{h}}{1+\frac{a}{h}}\left[\frac{1}{C^{3 / 2}}-i k a \frac{1}{C}\right] e^{i k_{\rho}} \cong \frac{1}{2 n} \frac{\frac{a}{h}}{1+\frac{a}{h}}\left[\left(\frac{a}{h}\right)^{3}\right. \\
\left.-i k a\left(\frac{a}{\rho}\right)^{2}\right] e^{i k \rho} \cong-\frac{1}{2 n} \frac{\frac{a}{h}}{1+\frac{a}{h}} i k a\left(\frac{a}{\rho}\right)^{2} e^{i k_{\rho}} .
\end{gathered}
$$

\section{Horizontal Top Loading}

In practice a horizontal or nearly horizontal top loading is often used. In this section the simplification which may be obtained in the formulas derived in the last section, as applied to the case of one or two wires when these wires are horizontal, will be considered, and a numerical calculation of the surface current will be carried out.

Setting $\alpha=\pi / 2$ the following expressions are obtained for the components of the normalized surface current density $\bar{j}^{h}$ induced by the horizontal wire

$$
\begin{aligned}
& j_{\rho}^{h}=-\frac{1}{2 \pi}\left(\frac{h}{a}\right)^{2} \cos (\phi-\beta), P \\
& j_{\phi}^{h}=\frac{1}{2 \pi}\left(\frac{h}{a}\right)^{2} \sin (\phi-\beta), P .
\end{aligned}
$$

From these formulas it is seen, as has also been shown before, that $\bar{j}^{h}$ is parallel to the horizontal wire. Therefore, $\bar{j}^{h}=j_{s}^{h} \hat{s}$ may be set, where $\hat{s}$ is a unit vector pointing in the positive direction of the wire. It is found that

$$
j_{s}^{h}=-\frac{1}{2 \pi}\left(\frac{h}{a}\right)^{2} P .
$$


The factor $P$ is expressed by the quantities $B, C, N$, and $X_{1}$ as in the last section. However, in the present case the expression for $B$ reduces to

$$
B=-\frac{\frac{\rho}{h}}{\frac{a}{h}} \cos (\phi-\beta) .
$$

The quantity $n\left|j_{s}^{h}\right|$ has been plotted in figures 8a and $8 \mathrm{~b}$ for $k h=0$ and in figures $9 \mathrm{a}$ and $9 \mathrm{~b}$ for $k h=0.1$, and in both cases for $\phi-\beta=0^{\circ}$ and $180^{\circ}$ as a function of $\rho / h$ and with $a / h$ as a parameter. From these curves and from similar curves for other values of $\phi-\beta$, the $\rho$ and the $\phi$ components of $n j^{h}$ by multiplying with $\cos (\phi-\beta)$ and $\sin (\phi-\beta)$, respectively, are obtained.

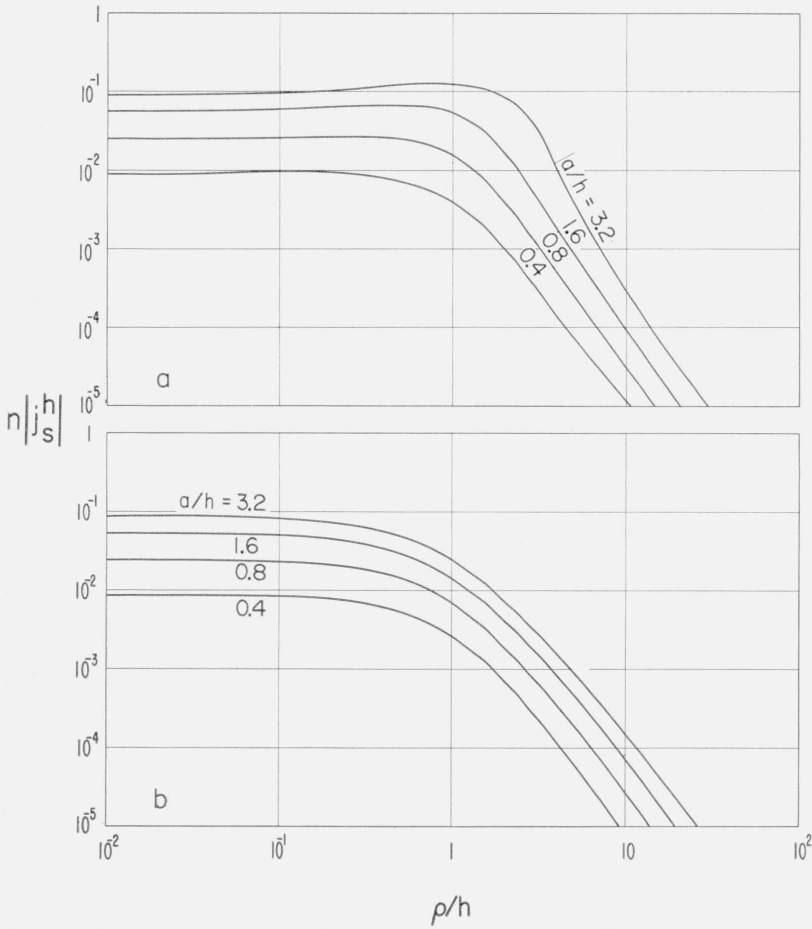

Figure 8. Normalized surface current density $\left|j_{s}^{h}\right|$ at the ground plane due to the current in the horizontal member of an antenna with one horizontal top-loading wire as a function of the normalized distance $\rho / h$ from the base of the antenna and with the normalized length $a / h$ of the top-loading wire as a parameter.

It is assumed that the height $h$ of the antenna is infinitely small compared to he wavelength, i.e, $k h=0$. a, $\phi-\beta=0^{\circ} ; \mathrm{b}, \phi-\beta=180^{\circ}$.

\section{L-Ântenna}

An L-antenna of the height $h$ and its horizontal member having the length $a$ as shown in figure 10 are considered. As in this case the number $n$ of wires in the top loading is equal to 1 , the contribution $\bar{j}^{h}$ to the normalized surface current density coming from the horizontal top loading may be obtained directly from figures 8 and 9 in the last section.

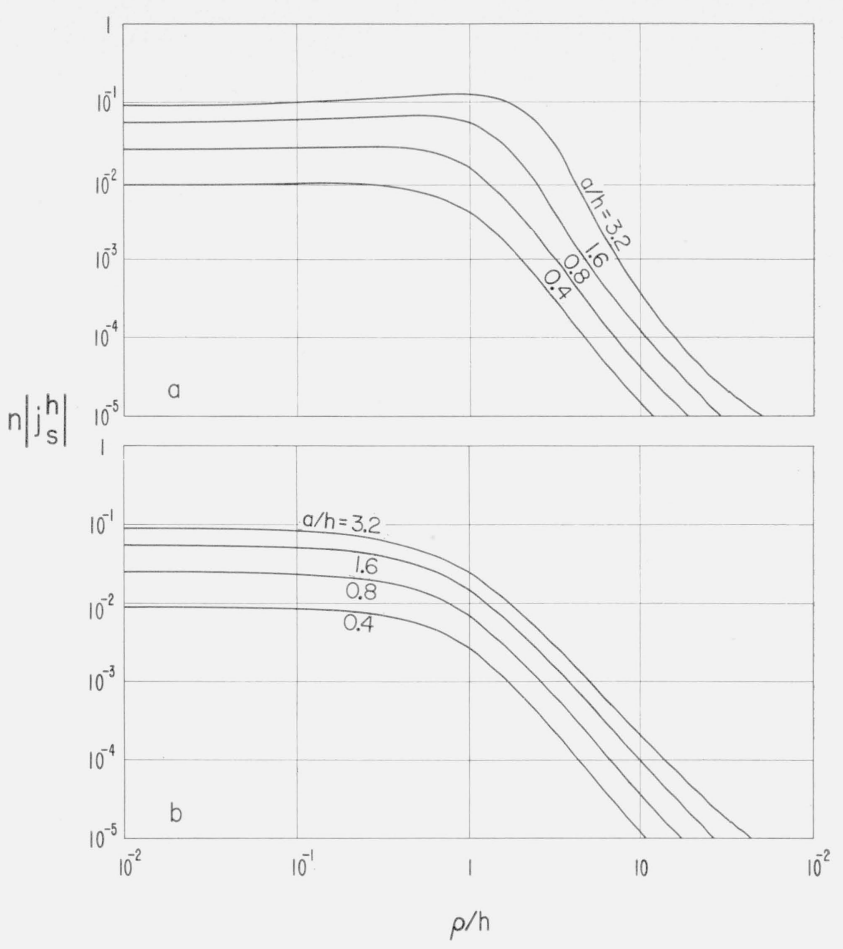

FIGURE 9. Normalized surface current density $\left|j_{s}^{h}\right|$ at the ground plane due to the current in the horizontal member of an antenna with one horizontal top-loading wire as a function of the normalized distance $\rho / h$ from the base of the antenna and with the normalized length $a / h$ of the top-loading wire as a parameter, the height $\mathrm{h}$ of the antenna being given by $\mathrm{kh}=0.1$.

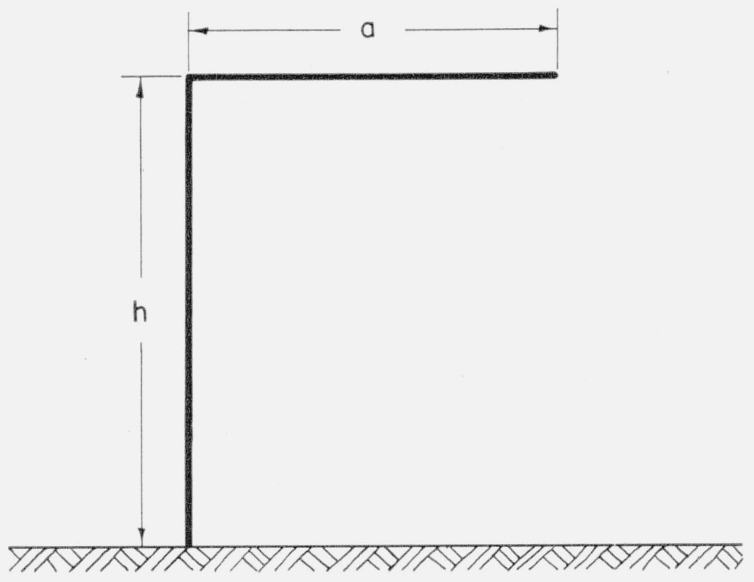

FigURE 10. L-antenna.

The contribution $\bar{j}^{h}$ obtained from these curves should be compared with the contribution $\bar{j}^{0}$ originating from the vertical member of the antenna as shown in figures 6 and 7 . A comparison of these figures shows that, compared to the radial component $j_{\rho}^{\circ}$ of the normalized surface current density induced by the vertical wire, the radial component $j_{\rho}^{h}$ of the normalized surface current density induced by the horizontal wire is small also in the vicinity of the 
antenna, and becomes insignificant at large distances. This was the same conclusion that was arrived at by Wait (see footnote 1) in his investigation of a diskloaded monopole. A similar statement may be made regarding the azimuthal component $j_{\phi}^{h}$ of the normalized surface current density induced by the horizontal wire. However, if the ground wires are placed radially around the antenna, as is usually the case, the ground losses induced by the azimuthal component $j_{\phi}^{h}$ may be significant compared to the losses induced by $j_{\rho}^{v}$, even if $j_{\phi}^{h}$ is considerably smaller than $j_{\rho}^{v}$.

In order to facilitate a comparison of $j_{\phi}^{h}$ with $j_{\rho}^{v}$, the quotient

$$
\left|\frac{j_{\phi}^{h}}{i_{\rho}^{v}}\right|=\frac{\left|j_{s}^{h} \sin (\phi-\beta)\right|}{\left|j_{\rho}^{v}\right|}
$$

has been plotted in figures $11 \mathrm{a}$ and $11 \mathrm{~b}$ for $k h=0$ and for $\phi-\beta=45^{\circ}$ and $135^{\circ}$. In the cases of $\phi-\beta=$ $0^{\circ}$ and $180^{\circ}$, respectively, the quotient is zero. Curves have not been given for the case of $k h=0.1$ treated earlier, as these curves would deviate only slightly from the curves in the case of $k h=0$ over the significant range of values of $\rho / h$.

\section{T-Antenna}

A T-antenna of the height $h$ and with its horizontal member having the length $2 a$ as shown in figure 12 is considered. Letting $\beta=\beta_{1}$ denote the angular position of one of the two horizontal wires of which the top loading consists, we have for the

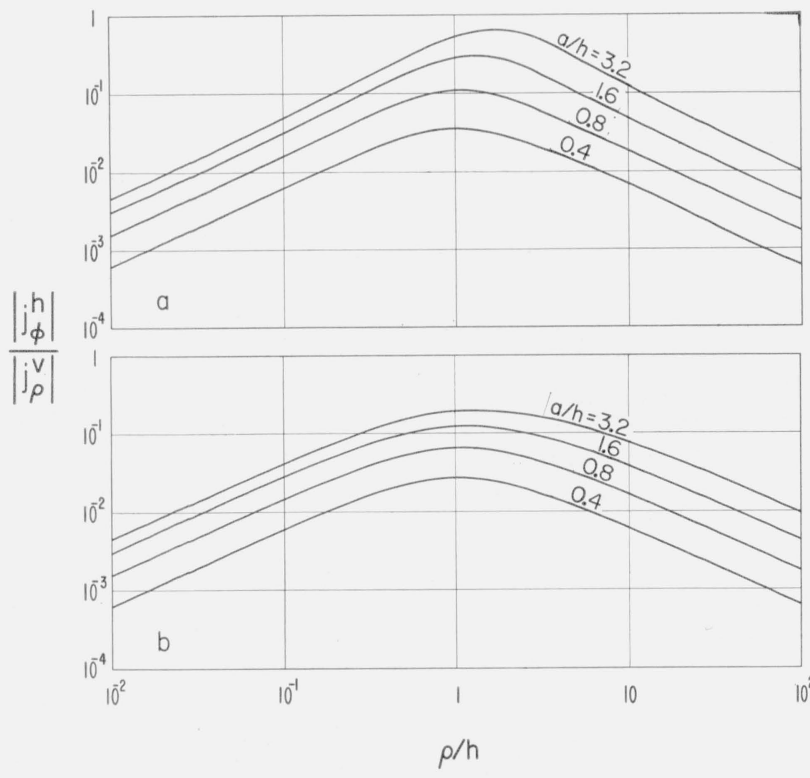

FIGURE 11. Ratio between the azimuthal component $\left|j_{\phi}^{h}\right|$ of the surface current density due to the current in the horizontal member of an L-antenna and the surface current density $\left|j_{\rho}^{v}\right|$ due to the vertical member of the same antenna.

It is assumed that the height $h$ of the antenna is infinitely small compared to the wavelength, i.e., $k h=0 ; \mathrm{a}, \boldsymbol{\phi}-\boldsymbol{\beta}=45^{\circ}$. b, $\boldsymbol{\phi}-\boldsymbol{\beta}=135^{\circ}$. angular position $\beta=\beta_{2}$ of the other radial wire $\beta_{2}=\beta_{1}+\pi$. Utilizing the symmetry properties of the normalized surface current density $\bar{j}^{h}$ induced by one radial wire it is then found that the following expressions for the normalized surface current density $\bar{j}^{H}$ of the total top loading by adding the contributions from each of the two wires,

$$
\begin{array}{r}
\bar{j}^{H}\left(\phi-\beta_{1}\right)=\bar{j}^{h}\left(\phi-\beta_{1}\right)+\bar{j}^{h}\left(\phi-\beta_{2}\right)=\hat{s}_{1} j_{s}^{h}\left(\phi-\beta_{1}\right) \\
+\hat{s}_{2} j_{s}^{h}\left(\phi-\beta_{1}-\pi\right),
\end{array}
$$

where $\hat{s}_{1}$ and $\hat{s}_{2}$ denote unit vectors in the positive directions of the two top-loading wires, respectively, as shown in figure 12 , and where $j_{s}^{h}(u)$ is the function introduced in the section on a general, horizontal top loading. Utilizing

$$
\hat{s_{2}}=-\hat{s}_{1}
$$

and

$$
j_{s}^{h}(-u)=j_{s}^{h}(u),
$$

it is found that

$$
\bar{j}^{H}\left(\phi-\beta_{1}\right)=\hat{s}_{1} j_{s_{1}}^{H}\left(\phi-\beta_{1}\right),
$$

where

$$
j_{s_{1}}^{H}\left(\phi-\beta_{1}\right)=j_{s}^{h}\left(\phi-\beta_{1}\right)-j_{s}^{h}\left(\pi-\left(\phi-\beta_{1}\right)\right) .
$$

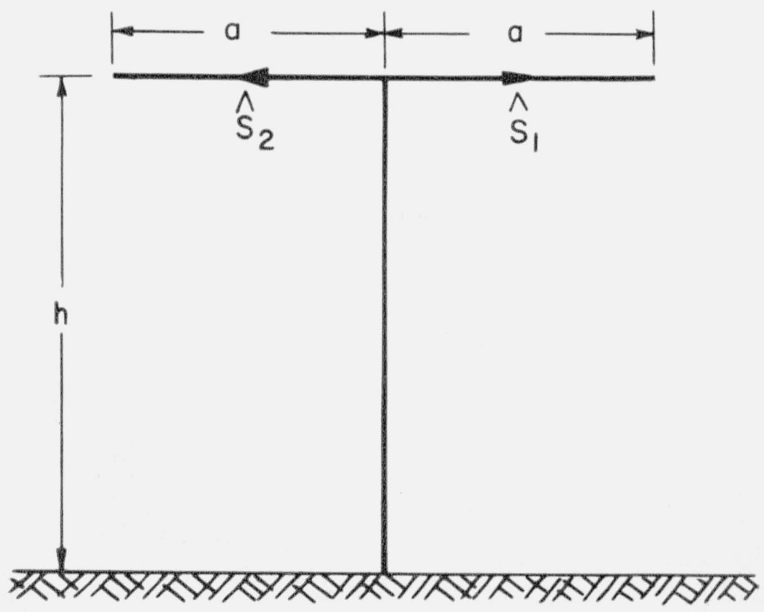

Figure 12. T-antenna.

As the number of wires in the top loading is equal to 2 , the function

$$
j_{s}^{h}\left(\phi-\beta_{1}\right)
$$

may be obtained as one-half the value given by the curves in figure 8 or 9.

It is easily verified that $j_{s}^{h}(\phi-\beta)$ satisfies the following symmetry relations,

$$
\begin{gathered}
j_{s_{1}}^{H}\left[-\left(\phi-\beta_{1}\right)\right]=j_{s_{1}}^{H}\left(\phi-\beta_{1}\right), \\
j_{s_{1}}^{H}\left[\pi-\left(\phi-\beta_{1}\right)\right]=j_{s_{1}}^{H}\left(\phi-\beta_{1}\right) .
\end{gathered}
$$


It follows from the above expression for $\bar{j}^{H}$ that

$$
\bar{j}^{H}\left( \pm \frac{\pi}{2}\right)=0 .
$$

Because of the symmetry relations mentioned above, it is necessary only to calculate the normalized surface current density for $\phi-\beta_{1}$ being in the interval 0 to $\pi / 2$.

The quantity $\left|\bar{j}_{s_{1}}^{H}(\phi-\beta)\right|$ has been plotted in figure 13 for $k h=0$ and for $\phi-\beta_{1}=0^{\circ}$ as a function of $\rho / h$ and with $a / h$ as a parameter. This diagram giving the contributions to the normalized surface current density arising from the horizontal wire should be compared to the contributions from the vertical member shown in figures 6 and 7 . This comparison leads to the same qualitative statements regarding the significance of the relative contributions from the horizontal wire as were arrived at in the case of the L-antenna.

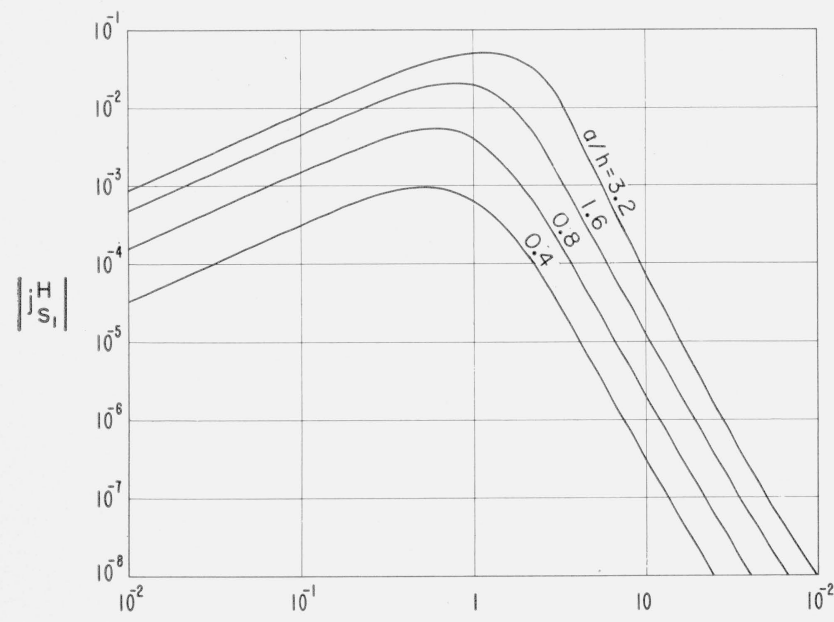

FIGURE 13. Normalized surface current density $\left|j_{s_{1}}^{-H}\right|$ at the ground plane due to the current in the horizontal members of a T-antenna as a function of the normalized distance $\rho / h$ from the base of the antenna and with the normalized length a/h of the top-loading wires as a parameter.

It is assumed that the height $h$ of the antenna is infinitely small compared to the wavelength, i.e., $k h=0 ; \phi-\beta=0^{\circ}$.

In order to be better able to survey the situation, in figure 14 the following quotient is plotted,

$$
\left|\frac{j_{\phi}^{H}}{j_{\rho}^{v}}\right|
$$

for $k h=0$ and for $\phi=45^{\circ}$ as a function of $\rho / h$ and with $a / h$ as a parameter. For $\phi=0^{\circ}$ and $90^{\circ}$ the quotient is zero. Paying attention to this fact and comparing figure 14 with figures $11 \mathrm{a}$ and $11 \mathrm{~b}$, it can probably be said that in general the azimuthal component of the surface current density induced by the top loading is smaller for a T-antenna with a horizontal wire of the length $2 a$ than for an L-antenna of the same height and with a horizontal wire of the length $a$.

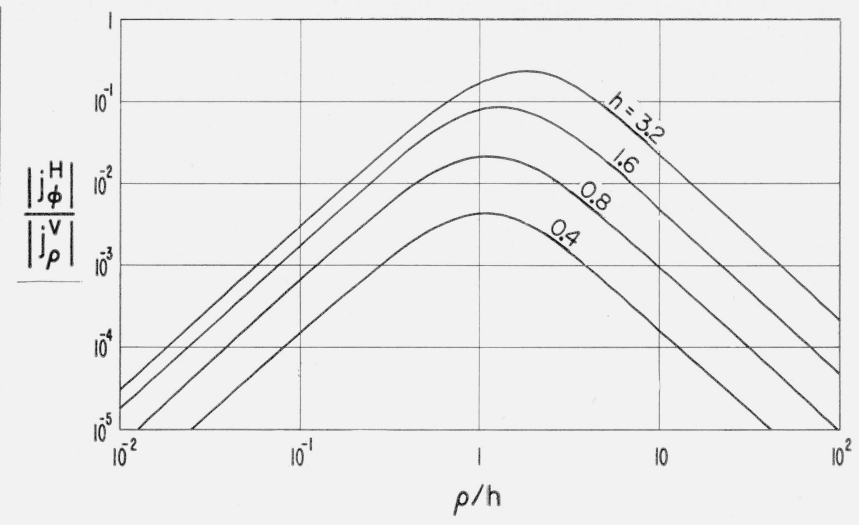

FiguRe 14. Ratio between the azimuthal component $\left|j_{\phi}^{h}\right|$ of the surface current density due to the current in the horizontal members of a T-antenna and the surface current density $\left|j_{\rho}^{v}\right|$ due to the vertical member of the same antenna.

It is assumed that the height $h$ of the antenna is infinitely small compared to the wavelength, i.e., $k h=0$. $\phi-\beta=45^{\circ}$.

On the other hand, the radial component will at some points be larger, at some points smaller for the T-antenna than for the corresponding $L$-antenna. For an antenna with a radial ground wire system it is essential to keep the azimuthal component of the surface current density as small as possible in order to make the total ground losses the smallest possible. In this respect a T-antenna is probably in general preferable to an L-antenna.

\section{Antenna with Four Horizontal Top-Loading Wires}

The theory developed above for the surface current induced on the ground plane by a straight wire with a linear current distribution will be used also for finding the earth currents induced by a toploaded antenna with four equiangularly spaced wires in the top loading. Although in the present case the assumption of a linear current distribution, as was mentioned before, probably does not approximate the actual current distribution so well as in the case of an L- or a T-antenna, it is desirable to include the case of an antenna with four horizontal top-loading wires, as this case is very close to the case of a disk-loaded antenna; on the basis of the results obtained in this section it should therefore be possible to make a comparison between the theory developed in this report and the theory developed for a disk-loaded monopole. However, it should be kept in mind that whereas the formulas and numerical results obtained in this report are based on the assumption of a linear current distribution on the top loading, Wait in his investigation of a disk-loaded antenna assumes a quadratic current distribution on the disk.

The length of the vertical member of the antenna is denoted by $h$ and the length of the top-loading wires by $a$ as indicated in figure 15. Further, the azimuth of the top-loading wires is denoted by $\beta_{1}$, $\beta_{2}, \beta_{3}$, and $\beta_{4}$, respectively, and the unit vectors 


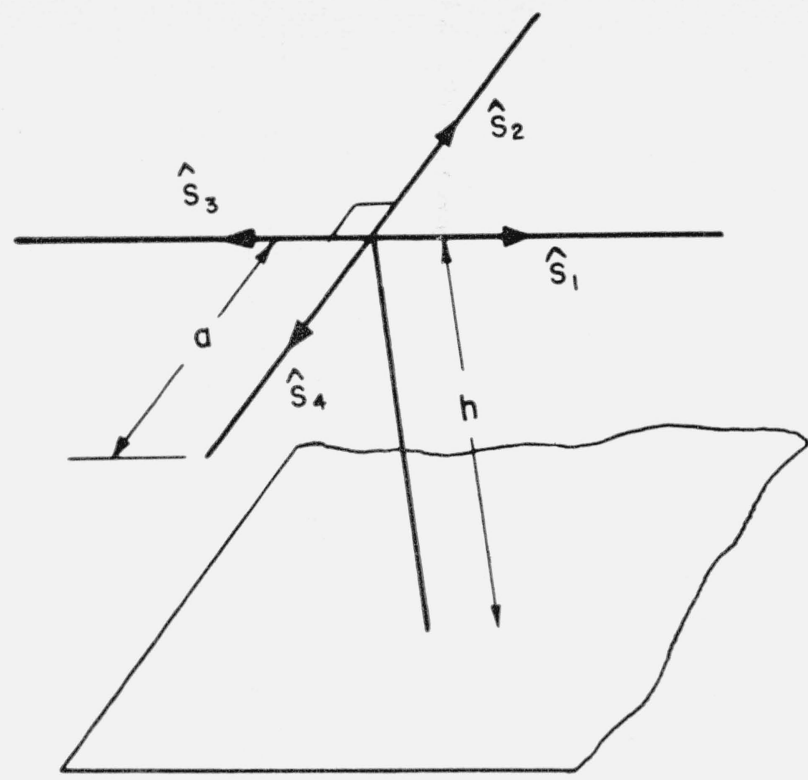

Figure 15. Antenna with four horizontal, top-loading wires.

pointing in the positive direction of these wires by $\hat{s}_{1}, \hat{s}_{2}, \hat{s}_{3}$, and $\hat{s}_{4}$. Then,

$$
\begin{aligned}
& \beta_{2}=\beta_{1}+\frac{\pi}{2}, \\
& \beta_{3}=\beta_{1}+\pi, \\
& \beta_{4}=\beta_{1}+\frac{3 \pi}{2},
\end{aligned}
$$

and

$$
\begin{aligned}
& \hat{s}_{3}=-\hat{s}_{1}, \\
& \hat{s}_{4}=-\hat{s}_{2} .
\end{aligned}
$$

Making use of the symmetry properties of the normalized surface current density $\bar{j}^{h}$ induced by one radial wire it is found that the following expressions for the normalized surface current density $j^{+}$of the total top loading:

$$
\begin{gathered}
\bar{j}^{+}\left(\phi-\beta_{1}\right)=\hat{s}_{1} j_{s}^{h}\left(\phi-\beta_{1}\right)+\hat{s}_{2} j_{s}^{h}\left(\phi-\beta_{2}\right) \\
+\hat{s}_{3} j_{s}^{h}\left(\phi-\beta_{3}\right)+\hat{s}_{4} j_{s}^{h}\left(\phi-\beta_{4}\right) \\
=\hat{s}_{1} j_{s_{1}}^{+}\left(\phi-\beta_{1}\right)+\hat{s}_{2} j_{s_{2}}^{+}\left(\phi-\beta_{1}\right),
\end{gathered}
$$

where

$j_{s_{1}}^{+}\left(\phi-\beta_{1}\right)=j_{s}^{h}\left(\phi-\beta_{1}\right)-j_{s}^{h}\left(\pi-\left(\phi-\beta_{1}\right)\right)$,

$j_{s_{2}}^{+}\left(\phi-\beta_{1}\right)=j_{s}^{h}\left(\frac{\pi}{2}-\left(\phi-\beta_{1}\right)\right)-j_{s}^{h}\left(\phi-\beta_{1}+\frac{\pi}{2}\right)$.
It may be verified that $\bar{j}^{+}\left(\phi-\beta_{1}\right)$ satisfies the following symmetry relations:

$$
\begin{gathered}
\bar{j}^{+}\left(-\left(\phi-\beta_{1}\right)\right)=\hat{s}_{1} j_{s_{1}}^{+}\left(\phi-\beta_{1}\right)-\hat{s}_{2} j_{s_{2}}^{+}\left(\phi-\beta_{1}\right), \\
\bar{j}^{+}\left(\frac{\pi}{2}-\left(\phi-\beta_{1}\right)\right)=\hat{s}_{1} j_{s_{2}}^{+}\left(\phi-\beta_{1}\right)+\hat{s}_{2} j_{s_{1}}^{+}\left(\phi-\beta_{1}\right), \\
\bar{j}^{+}\left(\pi-\left(\phi-\beta_{1}\right)\right)=-\hat{s}_{1} j_{s_{1}}^{+}\left(\phi-\beta_{1}\right)+\hat{s}_{2} j_{s_{2}}^{+}\left(\phi-\beta_{1}\right) .
\end{gathered}
$$

It follows from the above expression for $\bar{j}^{+}$that

$$
\begin{array}{r}
\bar{j}^{+}(0)=\hat{s}_{1}\left[j_{s}^{h}(0)-j_{s}^{h}(\pi)\right]=\hat{\rho}\left[j_{s}^{h}(0)-j_{s}^{h}(\pi)\right], \\
\bar{j}^{+}\left(\frac{\pi}{4}\right)=\left(\hat{s}_{1}+\hat{s}_{2}\right)\left[j_{s}^{h}\left(\frac{\pi}{4}\right)-j_{s}^{h}\left(\frac{3 \pi}{4}\right)\right] \\
=\hat{\rho} \sqrt{2}\left[j_{s}^{h}\left(\frac{\pi}{4}\right)-j_{s}^{h}\left(\frac{3 \pi}{4}\right)\right],
\end{array}
$$

where $\hat{\rho}$ denotes a unit vector pointing in the radial direction. As the number $n$ of wires in the top loading is equal to 4 , the function

$$
j_{s}^{h}(\phi-\beta)
$$

may be obtained as one-fourth the value given by the curves in figure 8 or 9 .

Because of the above relations the whole current field will be known, if it has been calculated in the angular space

$$
0 \leqq \phi-\beta_{1}<\frac{\pi}{4}
$$

Using the symmetry relations stated above it is found that $\bar{j}^{+}\left(\phi-\beta_{1}\right)$ is purely radial for

$$
\phi-\beta_{1}=p \frac{\pi}{4}
$$

where $p$ is an integer. For these angles the radial component

$$
j_{\rho}^{+}\left(\phi-\beta_{1}\right)
$$

is given by

$$
\begin{aligned}
j_{\rho}^{+}\left(\phi-\beta_{1}\right) & \left\{\begin{array}{l}
j_{s}^{h}(0)-j_{s}^{h}(\pi) \text { for } \phi-\beta_{1}=q \frac{\pi}{2} \\
\sqrt{2}\left[j_{s}^{h}\left(\frac{\pi}{4}\right)-j_{s}^{h}\left(\frac{3 \pi}{4}\right)\right] \text { for } \phi-\beta_{1}=\frac{\pi}{4}+q \frac{\pi}{2}
\end{array}\right\}
\end{aligned}
$$

where $q$ is an integer.

The quantity $\left|j_{\rho}^{+}\left(\phi-\beta_{1}\right)\right|$ has been plotted in figure 16 for $k h \cong 0$ and for $\phi-\beta_{1}=0^{\circ}$ as a function of $\rho / h$ and with $a / h$ as a parameter. These curves should be compared with the curves given in Wait's report 
(see footnote 1) on the earth currents induced by the disk in a disk-loaded monopole. It is seen that the currents in figure 16 have the same trend and the same order of magnitude as the curves computed by Wait.

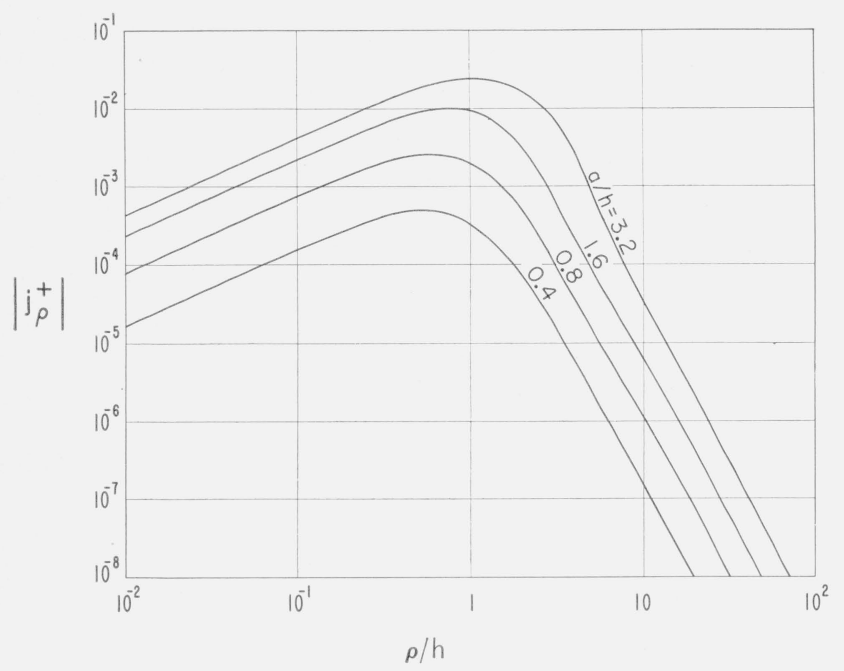

FIGURE 16. Radial component $\left|j_{\rho}^{+}\right|$of normalized surface current density due to the current in the horizontal members of an antenna with four horizontal, top-loading wires as a function of the normalized distance $\rho / h$ from the base of the antenna and with the normalized length $a / h$ of the top-loading wires as a parameter.

It is assumed that the height $h$ of the antenna is infinitely small compared to the wavelength, i.e., $k h=0 ; \phi-\beta=0^{\circ}$.

\section{Conclusion}

Based on a formula for and numerical calculations of the surface current density on a ground plane above which is placed a straight piece of wire with a length that is small compared to the wavelength and with a linear current distribution, the contribution to the ground current of the top loading of $L$ - and T-antennas that are small compared to the wavelength has been found and plotted. Whereas the contribution from the top-loading to the radial component of the surface current density, as was shown earlier by Wait, in general may be neglected, as it is insignificant compared to the contribution from the vertical wire, the azimuthal component of the surface current density produced by the top loading may be important in calculating the ground losses in the case of a system of radial ground wires, as it is perpendicular to these wires. 'The formulas and numerical results in this report form a basis for calculating the ground losses due to this azimuthal component.

This investigation was carried out while the author was temporarily employed as a consultant at the National Bureau of Standards in Boulder. $\mathrm{He}$ thanks the Bureau for the opportunity to do this work. He also thanks James R. Wait for suggesting the problem and Maxine W. Clark for making the numerical calculations.

Boulder, Colo., January 27, 1959. 